\title{
Bifurcation of the metric type II radio emission associated with the giant solar flare of April 22001
}

\author{
S. Mancuso and L. Abbo \\ Istituto Nazionale di Astrofisica (INAF), Osservatorio Astronomico di Torino, Strada Osservatorio 20, \\ Pino Torinese 10025, Italy \\ Received 3 December 2003 / Accepted 5 January 2004

\begin{abstract}
On April 2 2001, following one of the largest flares of the present solar cycle, intense and complex metric type II radio bursts were recorded by the Hiraiso Radio Spectrograph. Metric type II emission is a typical signature of the propagation of a coronal shock. The dynamic spectra of this event present a clear bifurcation of the radio emission lanes, which is very rare in the metric domain and requires a proper interpretation. We model this bifurcation as the result of the interaction of a pistondriven spherical shock with a vertical current sheet located above the active region where the X20 flare occurred. The geometry of the expanding transient and the coronal electron density, necessary to derive the type II dynamics, are constrained through analysis of data obtained from the Solar and Heliospheric Observatory (SOHO) instruments. The model replicates successfully the observed bifurcation, supporting our interpretation. Furthermore, we propose that magnetic reconnection was triggered in the current sheet by the shock passage.
\end{abstract}

Key words. Sun: corona - UV radiation - radio radiation - coronal mass ejections - shock waves

\section{Introduction}

The X20 solar flare that occurred on April 22001 at 21:51 UT above the huge active region NOAA 9393 (at N17 W67), was accompanied by a very fast $\left(>2000 \mathrm{~km} \mathrm{~s}^{-1}\right)$ coronal mass ejection (CME). Fast transients are expected to travel at supermagnetosonic speeds in the middle corona, thus developing piston-driven shock surfaces. Coronal shocks are detected in dynamic spectra as metric type II burst radiation: the propagation of a collisionless shock in the corona excites Langmuir waves which are then converted into electromagnetic radiation at frequencies near the local electron plasma frequency $f_{\mathrm{pe}}[\mathrm{Hz}]=8978 \sqrt{n_{\mathrm{e}}\left[\mathrm{cm}^{-3}\right]}$ and its second harmonic, where $n_{\mathrm{e}}$ is the electron density. An overview of the complex radio emission associated with the event studied in this work, is displayed in the dynamic spectrum obtained by the Hiraiso Radio Spectrograph (Fig. 1). A strong negatively drifting type II fundamental/harmonic pair is visible between 30 and $100 \mathrm{MHz}$. It starts from 21:52 UT lasting for about $5 \mathrm{~min}$ and drifting slowly toward lower frequencies. These emission lanes are the signatures of an expanding shock surface travelling through coronal plasma characterized by an outwardly decreasing electron density. The dynamic spectrum also shows an unexpected spectral feature: a strong type II

Send offprint requests to: $\mathrm{S}$. Mancuso,

e-mail: mancuso@to.astro.it positively drifting fundamental/harmonic pair lasting for about one minute starting at 21:51:30 UT between 100 and $300 \mathrm{MHz}$ that would imply a sunwardly propagating shock. In the past there have been only few identifications of positively drifting metric type II bursts (Markeev et al. 1976; Korolev et al. 1979), while more recently similar features were identified in the decametric (van der Holst et al. 2002) and decimetric bands (Huang 2003). The presence of such a rare spectral signature deserves special attention and requires an ad hoc interpretation. In the following sections we will show that the observed bifurcation of metric type II emission could have been produced by the interaction of a piston-driven spherical shock with a vertical current sheet located above active region NOAA 9393.

\section{Data analysis and modelling}

From the difference images of the Large Angle and Spectrometric Coronagraph (LASCO; Brueckner et al. 1995) aboard the Solar and Heliospheric Observatory (SOHO) spacecraft we derive the morphology of the expanding CME front surface (Fig. 2). The roughly circular shape observed by LASCO is interpreted as the sky-plane projection of a spherical plasmoid expanding from the solar surface and driving a bubble-type bow/piston shock surface. The axis of symmetry of the expanding bubble does not coincide with the streamer axis (compare left and center panels of Fig. 2) but they are separated by an angle $\theta$, left as a free parameter of the 


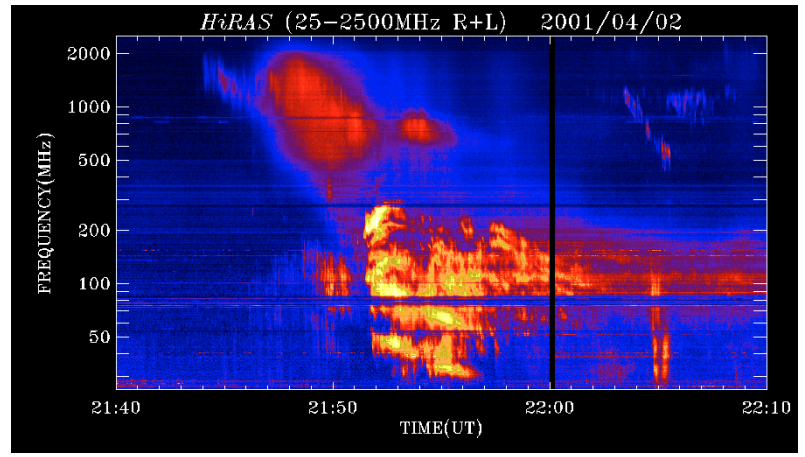

Fig. 1. Dynamic spectrum obtained by the Hiraiso radio spectrograph on April 22001 during the period 21:40-22:10 UT.

model (see cartoon on the top right of Fig. 3). Note that the angle between the two axes inferred from Fig. $2\left(\simeq 20^{\circ}\right)$ is a lower limit of $\theta$ due to projection effects. The height-time evolution of the CME leading edge observed by LASCO C2/C3 is shown (squares) together with a linear fit (dotted line) in the left panel of Fig. 3. The goodness of the fit is supported by the detection at $2.2 R_{\odot}$ of the CME by the Ultraviolet Coronagraph Spectrometer (UVCS; Kohl et al. 1995) aboard SOHO and by the start of a decimetric Drifting Pulsating Structure (DPS) (say, at $1.1 R_{\odot}$ ) visible after $21: 44$ UT in the dynamic spectrum, that could have been generated at the time of plasmoid ejection (Khan et al. 2002). We also show the onset (21:32 UT) and the peak (21:51 UT) of the flare. The location of the type II emission at a given frequency corresponds to the intersection of the shock wave with a particular density level (i.e., height) of the streamer. In the right panel of Fig. 3, the white dotted arrows point to the fundamental/harmonic frequencies emitted at the two intersections of the expanding shock surface with the streamer axis. The intersection points move, as a function of time, in opposite directions (as specified by the black arrows). Therefore, the dynamics of the type II emission can be deduced from the pre-shock coronal density profile $n_{\mathrm{e}}(r) \propto f_{\mathrm{pe}}(r)^{2}$, where $r$ is the height in solar radii (e.g. Mancuso et al. 2003).

\subsection{Pre-shock coronal density profile}

The pre-event coronal electron density of the streamer located above NOAA 9393 is estimated from synoptic observations performed by UVCS. Information on the coronal plasma density is inferred from the intensity and the profile of the brightest coronal lines emitted in the ultraviolet region via collisions and resonance scattering. The spectroscopic technique developed by Antonucci et al. (2004) provides a means to calculate the electron density on the basis of the doublet lines emitted from the same ion O VI at $\lambda 1031.93$ and $1037.62 \AA$. The electron density is derived from the ratio of collisional to radiative component of one of the two spectral lines and depends on the outflow velocity, since the expansion of the corona induces a Doppler dimming of the resonant scattering component of the line intensity. The electron density values inferred from the above diagnostics are nicely fitted by a power law: $n_{\mathrm{e}}(r)=1.35 \times 10^{5+\frac{3.3}{r}}$. On the same day, Manoharan \& Kundu (2003) reported the observation of a type II burst at 11:00 UT above active region NOAA 9393 with the Nancay Radioheliograph. These radio measurements showed that the $236 \mathrm{MHz}$ and $164 \mathrm{MHz}$ emissions (corresponding respectively to electron densities of $1.7 \times 10^{8}$ and $8.3 \times 10^{7} \mathrm{~cm}^{-3}$ for emission from the harmonic) were located respectively at 1.38 and $1.60 R_{\odot}$. The densities inferred from these observations have the same radial profile as the one estimated by the O VI doublet diagnostics except a multiplicative factor of 5.2. The above discrepancy in the values derived from the two different density diagnostics might be due to line-of-sight integration effects. In fact, on one hand, any thin structure, even much denser than the background density, would not produce significant contribution to the line-of-sight-averaged emission observed by UVCS. On the other hand, radio bursts are supposed to be selectively produced in the densest parts along streamer axes (e.g. Mancuso \& Raymond 2004), where the strength of fast-mode shocks can be very much enhanced. Vertical, dense and thin current sheets are expected to be present above the helmet structures in coronal streamers. From UVCS data, there is some observational evidence of current sheets through the detection of high ionization (high temperature formation) lines (Ciaravella et al. 2002; Ko et al. 2003). Unfortunately, the observed spectral range of UVCS data for the analysed event was not optimized for detection of these lines, therefore the presence of a current sheet must be inferred through other physical properties. By analysing the ultraviolet emission of the streamer axis above active region NOAA 9393, we found enhanced emission of the $\mathrm{H}$ I Ly $\alpha 1215.67 \AA$ line at $1.75 R_{\odot}$ together with an O VI $I(1032) / I(1038)$ doublet intensity ratio of $4.3 \pm 0.4$, much higher than expected at this height. Such a high doublet ratio seems to imply that the density is very low. However, this result is consistent with the fact that the our electron densities are derived by means of intensities that are integrated along the line of sight, therefore contribution of dense, thin plasma has to be negligible. Indeed, we estimate an upper limit of only 0.01 for the filling factor (evaluated as in Antonucci et al. 2004) of the dense structure. More important, the high value of the ratio indicates nearly static plasma (Noci et al. 1987) at $1.75 R_{\odot}$, which well represents the conditions in and just around a current sheet. In fact, if account is taken of the finite conductivity and the azimuthal magnetic field component of a coronal streamer, the field lines do not remain open but close across the sheet (Somov \& Verneta 1989). Therefore the velocity inside the sheet should be indeed very small due to the inhibiting effect of the transverse magnetic field (Pneuman 1972). Although the above properties are not conclusive, they strongly suggest the presence of a current sheet with density somewhat enhanced with respect to the average value of the streamer.

\subsection{Model results}

We model the shock front by using a spherical expanding surface as suggested by the LASCO images (Fig. 2). The bifurcation of the type II emission is reproduced with great accuracy for $\theta \simeq 27^{\circ}$ and enhancement factors in the range 5-5.5 relative to the density profile provided by UVCS, confirming the 

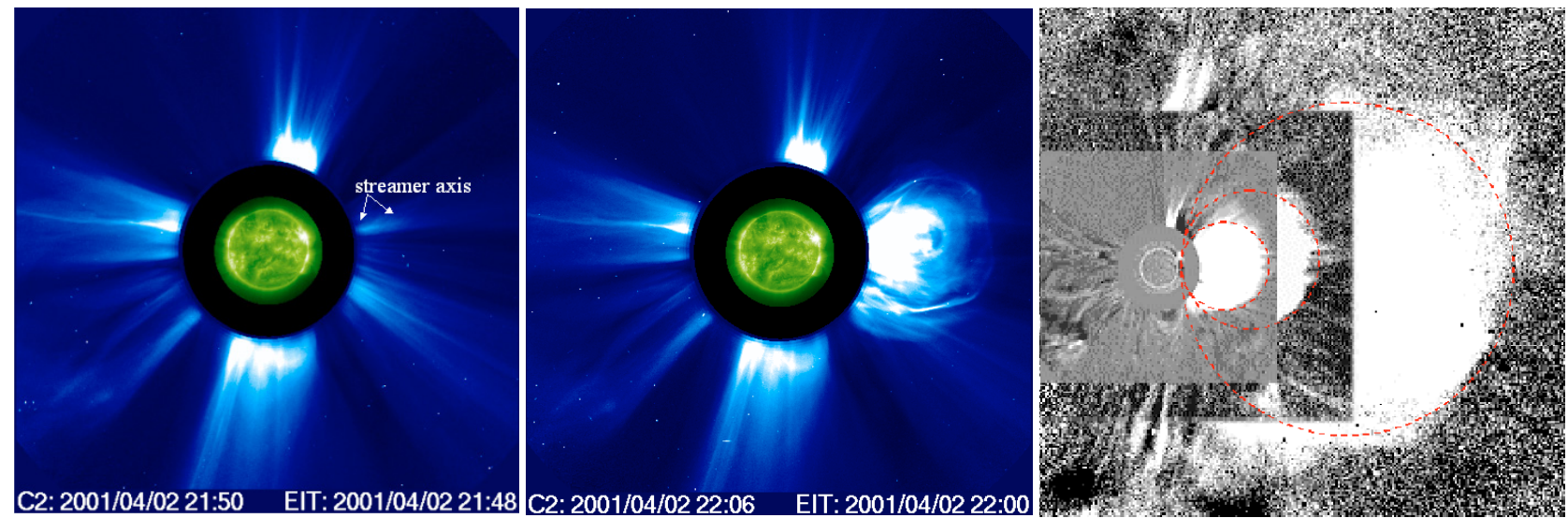

Fig. 2. Left panel: composite of images taken by two SOHO instruments at the time of the April 22001 solar flare: the inner image of the solar disk was taken by the Extreme ultraviolet Imaging Telescope (EIT; Delaboudiniere et al. 1995) in the Fe XII $195 \AA$ A line; the outer image of the corona was taken by the LASCO C2 coronagraph in visible light (times are specified below the images). Center panel: same as left panel but during the eruption. Right panel: LASCO C2/C3 difference images showing the eruption at three different times. The dashed curves trace the CME front surface in sky-plane projection.

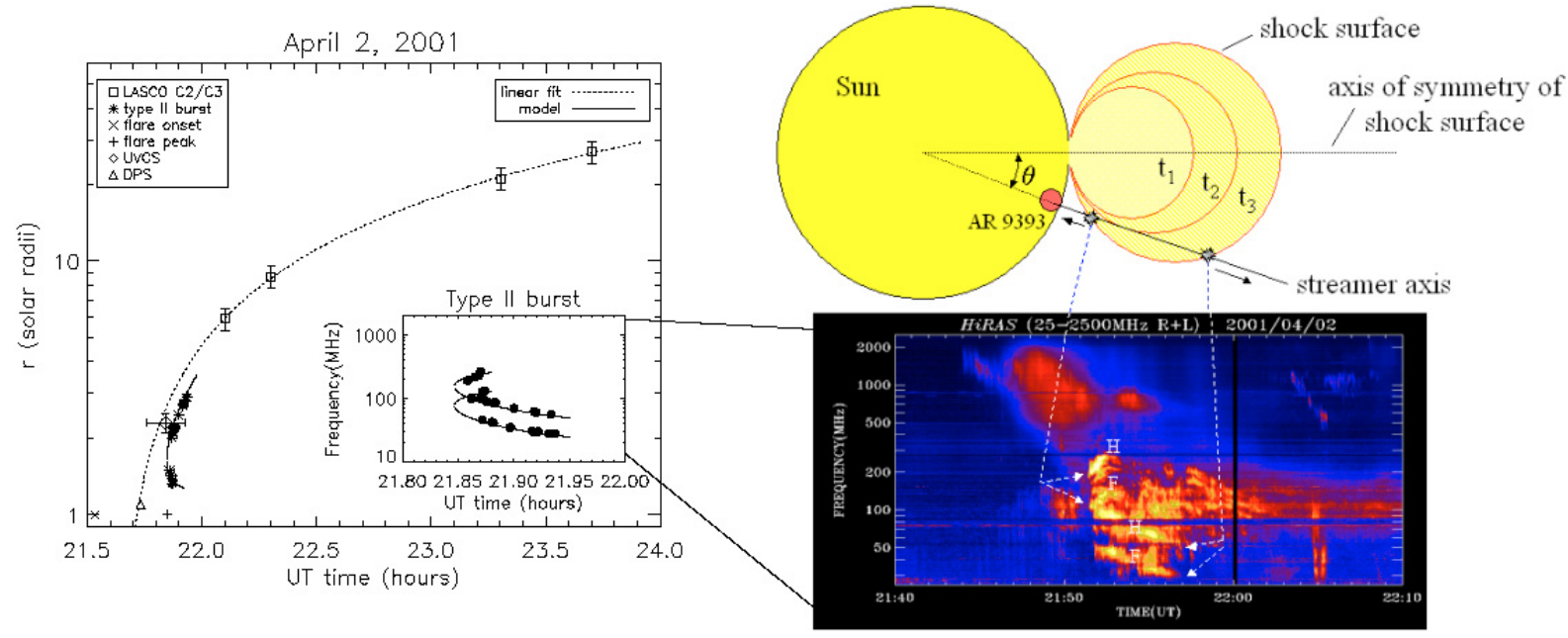

Fig. 3. Left panel: height-time evolution of the April 22001 CME (see text for details). The inset shows the fit derived from our model interpreting the bifurcation of the type II fundamental/harmonic pair. Top right panel: cartoon showing the scenario of the model for an observer located above the plane defined by the axis of symmetry of the expanding shock surface and the streamer axis at times $t_{1}<t_{2}<t_{3}$. Bottom right panel: same as Fig. 1.

presence of a dense current sheet unresolved with the O VI doublet diagnostics. The multiplicative factor that we derived from the analysis of the radio observations of Manoharan \& Kundu (2003) is remarkably within this range (see Sect. 2.1), meaning that density conditions above the active region were not dramatically changed after the previous event that occurred at $\sim 11: 00$ UT. In the inset of Fig. 3, we show the model fit (solid line) to the frequencies of the radio emission (filled circles) observed in the Hiraiso dynamic spectrum. For this plot the UVCS density profile is scaled by a factor of 5.2. In the same plot of the CME leading edge trajectory, the solid line marks the heights, derived by the model as a function of time, of the shock surface where the type II radiation is emitted. The type II radio emission heights (stars), obtained by converting the observed radio frequencies and using the adopted density profile, nicely overlap the model curve.

\section{Discussion and summary}

Our study strongly supports the idea that on April 2 2001, a curved piston-driven shock surface intersected a vertical current sheet located above active region NOAA 9393 at $\sim 21: 51 \mathrm{UT}$. It is tempting to investigate if the interaction of the shock with the current sheet could be also deduced indirectly through other signatures. In the following, we suggest a scenario for the subsequent development of the event and finally we describe the conclusions of our study.

\subsection{Shock-induced reconnection in the current sheet?}

The shock wave passage could trigger magnetic reconnection in a current sheet, for example through tearing-mode instability (e.g. Odstrčil \& Karlický 1997). In such a scenario, a new 


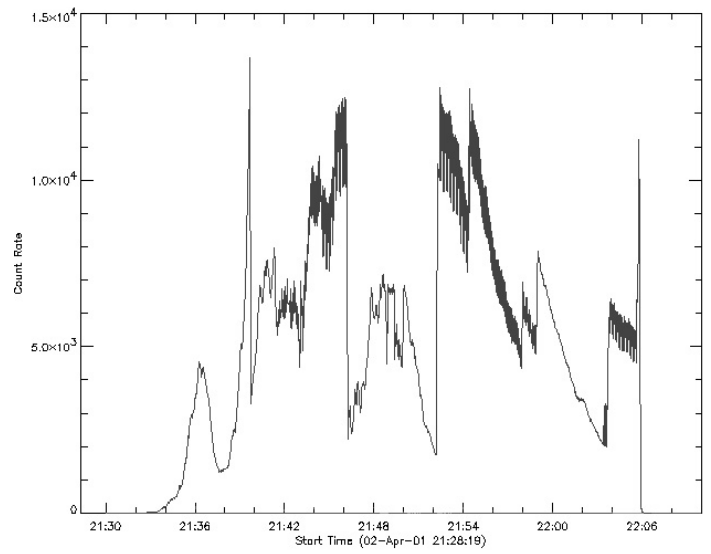

Fig. 4. Hard X-ray light curve of the event in the range 19-29 keV obtained by the Hard X-Ray Spectrometer (HXRS).

plasmoid located just above the reconnecting current sheet might be impulsively ejected from the corona. Recently, a zerodrift type II burst was interpreted by Aurass et al. (2002) as the radio signature of a termination shock in the reconnection outflow below an erupting prominence. Indeed, a second negatively drifting type II burst feature, visible from 21:57 UT and starting at about $200 \mathrm{MHz}$ (Fig. 1), could be generated by a different shock surface. This second shock would be driven by a supermagnetosonic plasmoid leaving active region NOAA 9393 after 21:51 UT, following shock-induced reconnection in the current sheet. The high emission frequencies of this second type II radio burst imply that the shock is propagating in the wake of the first CME, through already compressed plasma. Other fast drifting features in the metric dynamic spectrum that occurred after the bifurcation and a major broad peak observed in the hard X-ray emission at 21:54 UT (Fig. 4) (likely produced by thick-target emission of mildly relativistic electron beams) might also indicate impulsive particle acceleration due to this shock-induced reconnection stage.

\subsection{Conclusions}

A peculiar feature of the metric dynamic spectrum associated with the giant solar flare of April 22001 has been interpreted as due to bifurcation of the radio type II emission produced by a curved fast mode shock surface intersecting a vertical current sheet located above active region NOAA 9393. Although the model was oversimplified in that we assumed a constantly expanding spherical surface just above the transient, the results reproduce quite remarkably the observed bifurcation. The analysis of this event confirms that the electron densities sounded by the shock correspond to characteristic values of thin, enhanced density plasma structures, such as current sheets. Moreover, we suggest that the shock wave passage may have triggered magnetic reconnection just above active region NOAA 9393, causing the ejection of a second supermagnetosonic plasmoid after $\sim 21: 51 \mathrm{UT}$. Assuming that our interpretation for this event is correct, we speculate on the idea that radio emitting inward propagating shocks should occur more often than reported. Therefore, it would be worth to check for spectral signatures of bifurcation of type II emission for other very high speed transient events. Note, however, that the peculiar geometry of the expanding shock surface, needed to produce such a spectral feature, implies that the emission of the positively drifting lanes fades quickly: the speed of the inward propagating portion of the shock surface is likely to decrease quite strongly, soon becoming submagnetosonic. In general we expect that, if present, the bifurcation would be barely noticeable for a few tens of seconds and we suspect that this effect can be easily overlooked in routine data inspection.

Acknowledgements. We acknowledge the financial support of the Agenzia Spaziale Italiana. SOHO is a joint project of NASA/ESA. Hiraiso is operated by CRL, Japan. HXRS is a joint endeavor by the Academy of Sciences of the Czech Republic and the Space Environment Center of the NOAA.

\section{References}

Antonucci, E., Dodero, M. A., Giordano, S., Krishnakumar, V., \& Noci, G. 2004, A\&A, in press

Aurass, H., Vrsnak, B., \& Mann, G. 2002, A\&A, 384, 273

Brueckner, G. E., Howard, R. A., Koomen, M. J., et al. 1995, Sol. Phys., 162, 357

Ciaravella, A., Raymond, J. C., Li, J., et al. 2002, ApJ, 575, 1116

Delaboudiniere, J.-P., Artzner, G. E., Brunaud, J., et al. 1995, Sol. Phys., 162, 291

Huang, G. 2003, New Astronomy, 8, 213

Khan, J. I., Vilmer, V., Saint-Hilaire, P., \& Benz, A. D. 2002, A\&A, 388,363

Ko, Y., Raymond, J. C., Lin, J., et al. 2003, ApJ, 594, 1068

Kohl, J. L., Esser, R., Gardner, L. D., et al. 1995, Sol. Phys., 162, 313

Korolev, O. S., Fomichev, V. V., \& Chertok, I. M. 1979, Sov. Astron., 23, 216

Mancuso, S., Raymond, J. C., Kohl, J., et al. 2003, A\&A, 400, 347

Mancuso, S., \& Raymond, J. C. 2004, A\&A, 413, 363

Manoharan, P. K., \& Kundu, M. R. 2003, ApJ, 592, 597

Markeev, A. K., Formichev, V. V., \& Chertok, I. M., 1976, Sov. Astron., 20, 710

Noci, G., Kohl, J. L., \& Withbroe, G. L. 1987, ApJ, 315, 706

Odstrčil, D., \& Karlický, M. 1997, A\&A, 326, 1252

Pneuman, G. W. 1972, Sol. Phys., 23, 223

Somov, B. V., \& Verneta, A. I. 1989, Sol. Phys., 120, 93

van der Holst, B., Van Driel-Gesztelyi, L., \& Poedts, S. 2002, ESA SP-506, 71 